\title{
Consejo psicológico y psicoterapia motivacional en drogodependencias: evaluación de las intervenciones del programa "Lugar de Escucha"- CEDRO
}

\author{
Milton J. Rojas Valero'; Luis S. Espinoza Paul ${ }^{2}$ \\ 1 Jefe del Programa "Lugar de Escucha" del Centro de Información y Educación para la Prevención del Abuso de Drogas - CEDRO \\ 2 Psicólogo del Programa "Lugar de Escucha" del Centro de Información y Educación para la Prevención del Abuso de Drogas - CEDRO.

\section{RESUMEN}

La investigación tiene como objetivo la evaluación de la percepción y satisfacción, así como de las intervenciones breves a través del consejo psicológico y sesiones motivacionales del programa "Lugar de Escucha". El estudio es de tipo pre-experimental, con un diseño de medición pretest-postest de un solo grupo. La muestra estuvo constituida por 128 participantes de ambos sexos (9,4\% mujeres y $90,6 \%$ hombres), con edades comprendidas entre 15 y 51 años de edad (Media = 23,65; Desviación estándar = 7,92), consumidores de marihuana, pasta básica de cocaína, clorhidrato de cocaína, inhalantes y alcohol que asistieron al programa. La recolección de los datos se hizo a través de la Ficha de Atención (FdA); la Escala de Evaluación para el Cambio de la Universidad Rhode Island (URICA); el Cuestionario de Satisfacción con el Tratamiento (CST) y la Encuesta de Seguimiento de Casos (ESC). Para medir la eficacia del programa "Lugar de Escucha» se evaluaron los estadios motivacionales de los consultantes, tanto en acogida como en derivación, el grado de satisfacción con relación al servicio recibido y el porcentaje de inserción a los diferentes programas de tratamiento de los consultantes derivados.

Los resultados sobre percepción y satisfacción confirman el predominio de las fortalezas del programa. En lo que se refiere a las fases motivacionales, se encontró que las intervenciones de inducción motivacional mantienen y afianzan en el paciente la motivación de cambio de actitud y el nivel motivacional para interrumpir el consumo. De esta manera, de acuerdo con lo encontrado, dichas intervenciones tienden a incrementar su eficacia cuando los pacientes inician el proceso de tratamiento en los estadios de precontemplación y contemplación. Ello sugiere la necesidad de trabajar con muestras más homogéneas, considerando el tipo de drogas, edad y sexo, y la aplicación de instrumentos pre y post. Asimismo, lo encontrado sugiere la necesidad de clasificación de los pacientes en estadios de cambio; lo que puede ser una herramienta útil para el perfeccionamiento de los programas de tratamiento en drogodependencias.

Palabras clave: estadios motivacionales, satisfacción, consejería, drogodependencias.

\section{ABSTRACT}

The objective of the present research is to assess perception of and levels of satisfaction with the Lugar de Escucha program, as well as its brief interventions using counseling and motivational sessions. The study is of a pre-experimental type, with a single-group pretest-posttest measurement design. The sample was made up of 128 participants $(9.4 \%$ females and $90.6 \%$ males), aged 15 to 51 (mean= 23.65; standard deviation $=7.92$ ), users of cannabis, cocaine base paste, cocaine, inhalants and alcohol who attended the program. Data collection was carried out using Attention Forms (FdA); the University of Rhode Island Change Assessment Scale (URICA); the Treatment Satisfaction Questionnaire (CST); and the Cases Follow-up Survey (ESC). In order to evaluate the effectiveness of the Lugar de Escucha Program, we assessed participants' motivational phases during the first interview and at referral, their level of satisfaction with the service received and the percentage of entrance to different treatment programs of the referred participants.

The results on perception and satisfaction confirm a predominance of the program's strengths. With regard to the motivational phases, the findings show that the motivational induction interventions help to establish and maintain the patient's motivation for attitude change and for cessation of the abuse. In this sense, according to the findings, such interventions tend to be more effective when applied to patients in the Precontemplational and Contemplational phases. This suggests the need to work with more homogeneous groups, considering type of drugs, age and gender, and to use pre and post instruments. Likewise, the results suggest the need to classify patients in phases of change; such classification could be a useful tool for the improvement of treatment programs for drug users.

Key words: motivational phases, satisfaction, counseling, drug abuse. 


\section{NTRODUCCIÓN}

E la actualidad se ha generado la necesidad de evaluar la actividad de los servicios asistenciales en casos de drogodependencias. No escapan a esta realidad los servicios sociales y asistenciales en el Perú, donde se plantean problemas extremadamente complejos, ya que no es fácil definir el producto final, haciéndose necesario introducir el concepto de calidad, así como también la motivación e interés de los que quieren la evaluación (pacientes, políticos, gerentes, profesionales, aseguradoras, científicos, etc.).

Desde la perspectiva clínica podemos diferenciar los diversos tipos de consultantes, grupos etarios, género y estrato social, así como reconocer el grado de compromiso de cambio del consultante, y también los patrones de uso y abuso de sustancias psicoactivas (en adelante SPAs). Por lo cual surge la necesidad de confrontar los procesos de intervención que lleva a cabo nuestro programa, buscando consolidar y validar los más ventajosos. Si consideramos que en el Perú la evaluación sigue siendo una actividad poco practicada, y especialmente la referida al campo clínico de la dependencia de SPAs, concluiremos que el papel del investigador ha sido muy modesto.

Por otro lado, el interés por evaluar la satisfacción de los clientes adictos hacia los servicios de salud se ha incrementado considerablemente. Esto permitió hacer reformas en dichos servicios en años recientes. Aun siendo esporádico la evaluación, los escasos recursos y las constantes reformas de los servicios de salud no han permitido desarrollar el hábito de evaluar las intervenciones en adicciones. Sin embrago, los estudios sobre la satisfacción del cliente deben convertirse en un componente integral que permita crear estrategias de manejo y monitoreo de los programas de atención en salud (Bartu, 1996).

Es en Norteamérica donde se han llevado a cabo estudios de evaluación más amplios y sistemáticos, aunque ellos no examinaron el tema motivacional como predominante. Los estudios de evaluación clásicos en la bibliografía americana son los programas DARP (Drug Abuse Reportting Program), TOPS (Treatment Outcome Prospective Study) y DATOS (Drug Abuse Treatment Outcome Study).

Rumpf, Hapke y John (1998) investigaron en Alemania la relación entre los estadios de cambio y el pedido de ayuda inicial en un grupo de pacientes alcohólicos de un hospital general. Los resultados mostraron que un $10,9 \%$ se encontraba en estadio de precontemplación y un $84,8 \%$ estaba en estadio de contemplación o acción, lo cual contrasta con la opinión de los clínicos quienes manifestaron que esta población no estaba motivada para cambiar su com- portamiento de uso. Con estos datos se demostró la importancia de la intervención, teniendo en cuenta los estadios motivacionales en los que se encontraban los pacientes.

Santos et al, 2001, exploraron los estadios y procesos de cambio motivacionales en un grupo de 116 dependientes $(91,4 \%$ hombres y $8,6 \%$ mujeres) en tratamiento ambulatorio utilizando la Escala de Evaluación para el Cambio de la Universidad de Rhode Island (URICA) y el Inventario de Procesos de Cambio (IPC). El análisis de los datos encontró una predominancia del estadio acción $(51,7 \%)$ seguido del estadio precontemplación y contemplación $(30,1 \%)$. En relación con los procesos de cambio (liberación social y control de estímulos), la puntuación obtenida fue superior a la media de la muestra, concluyendo que la intervención psicológica debería dirigirse hacia el aumento de la atribución interna y capacidad de decisión sobre la conducta adictiva, así como a la reestructuración del ambiente.

Da Silva et al, 2003, evaluaron los estadios motivacionales en 120 alcohólicos y exploraron si existía una correlación entre estos estadios de cambio y el grado de alcoholismo. Se utilizó el Shortform Alcohol Dependence Data (SADD) para identificar la gravedad del alcohólismo y la Escala de Evaluación para el Cambio de la Universidad de Rhode Island (URICA) para evaluar los estadios motivacionales. Los resultados encontraron una correlación positiva y de intensidad baja $(r s=0,197 ; p=0,031)$ entre la dependencia alcohólica y el estadio de mantenimiento. Las conclusiones dan a conocer la importancia de la aplicación de las escalas para realizar mejoras en los lineamientos de la psicoterapia.

En Brasil, Da Silva (2005a), realizó un estudio con 109 drogodependientes. En los resultados verificó un aumento significativo en el estadio de mantenimiento $(p<0,001)$ dentro del tratamiento. Fueron evaluados 70 dependientes de cocaína internados. Al aplicar la Escala de Evaluación para el Cambio de la Universidad de Rhode Island (URICA) se percibieron los promedios para precontemplación $(17,03)$, contemplación $(35,63)$, acción $(35,10)$ y mantenimiento $(33,33)$, demostrándose que en el momento del internamiento se encontraban en la fase de acción, a pesar de presentar una puntuación alta en la etapa precontemplativa. Ello sugiere que los consumidores no percibían que la cocaína era un problema en sus vidas.

Da Silva (2005b) estudio a 39 adolescentes consumidores de marihuana a través de entrevistas motivacionales e intervención breve. Para los efectos se utilizo la Escala de Evaluación para el Cambio de la Universidad de Rhode Island (URICA). El 84\% de los participantes se situó en el estadio precontemplativo, 
ello permitió verificar cambios significativos en la fase de precontemplación dado que los adolescentes mostraron mayor conciencia del problema adictivo.

Haciendo un balance de los estudios revisados, podemos decir que la evaluación de los estadios de cambio en drogodependencias permite hacer una exploración multidimensional de la conducta adictiva y también desarrollar los objetivos. Así, explora y determina los estadios por los que se desplaza la motivación de cambio en forma de espiral. Del mismo modo, la evaluación de la satisfacción con el tratamiento ofrece la posibilidad de realizar cambios favorables en los programas de tratamiento y mejorar el entrenamiento de los profesionales en el campo de las adicciones.

Los siguientes cinco estadios de cambio constituyen la estructura o dimensión temporal o evolutiva del modelo transteórico: precontemplación, contemplación, preparación, acción y mantenimiento.

Estos estadios representan, pues, los aspectos temporales y motivacionales del cambio intencional. Dicho cambio no es un fenómeno de todo o nada, sino un movimiento gradual a través de etapas específicas, donde cada uno representa tanto un lapso así como una serie de habilidades y tareas necesarias para pasar a la siguiente etapa. El tiempo en cada una de estas fases puede variar, mientras que las tareas son realizadas en orden para conseguir el movimiento exitoso al siguiente espacio (Prochaska et al, 1991)

No obstante, y dado que uno de los principales problemas en el proceso de cambio de cualquier conducta adictiva es el de las elevadas tasas de recaída (Casas y Gossop, 1993; Marlatt y Gordon, 1985), el modelo transteórico resalta el hecho de que la mayoría de las personas no progresan linealmente a través de los estadios de cambio. (Prochaska et al, 1991).

Por ello, conceptualizar los estadios mediante un modelo en espiral y dinámico, a diferencia del modelo lineal, refleja con mayor fidelidad el proceso de cambio en el campo de las conductas adictivas.

Lugar de Escucha de CEDRO asume como psicoterapia motivacional a un tipo de abordaje psicoterapéutico breve con un marco cognitivo-conductual y humanista (Adaptación de la Motivational enhancement therapy o psicoterapia de estimulación motivacional), probado por el CYT Cannabis Youth Treatment), el SAMHSA (Substance Abuse and Mental Health Services Administrationn) y el CSAT (Center for Substance Abuse Treatment) en pacientes adolescentes consumidores de marihuana.

\section{OBJETIVOS DEL ESTUDIO}

\section{Objetivo general:}

- Determinar la eficacia del consejo psicológico y de las sesiones motivacionales proporcionadas en casos de drogodependencias por el programa "Lugar de Escucha» de CEDRO.

\section{Objetivos específicos:}

- Determinar los estados motivacionales de los consultantes, tanto en acogida como en derivación.

- Explorar la evolución motivacional en los consultantes luego de las sesiones de consejería, orientación y motivación.

- Evaluar el porcentaje de inserción a los diferentes programas de tratamiento de los consultantes derivados.

- Examinar el grado de satisfacción de los consultantes con relación al servicio recibido.

\section{MÉTODOS}

\section{Diseño de investigación}

El tipo de diseño utilizado es pre-experimental, con una medición pretest-postest de un solo grupo, lo cual permitió analizar el cambio antes y después de los consultantes al programa "Lugar de Escucha» de CEDRO en relación con las variables estudiadas (Kerlinger, 1994).

\section{Participantes}

El muestreo utilizado es no-probabilístico e intencional. La muestra está constituida por 128 participantes voluntarios de ambos sexos $(9,4 \%$ mujeres y $90,6 \%$ hombres), con edades comprendidas entre los 15 y 51 años de edad (media = 23,65; desviación estándar $=7,92$ ). Los colaboradores del estudio son consumidores de diversas SPA (marihuana, pasta básica de cocaína, clorhidrato de cocaína, inhalantes y alcohol) que solicitaron asistencia al programa de consejería y orientación psicológica "Lugar de Escucha» de CEDRO. Se excluyeron del estudio los consultantes que presentaban policonsumo, trastornos psiquiátricos asociados al consumo o signos de intoxicación por SPA durante la entrevista.

\section{Instrumentos}

Para la recolección de datos demográficos y de consumo de drogas se utilizo la Ficha de Atención -FdA (Rojas, Giraldo y Montes, 2001). 
Para el diagnóstico de los estadios motivacionales se aplico la Escala de Evaluación para el Cambio de la Universidad de Rhode Island - URICA (McConnaughy, Prochaska y Velicer, 1983; McConnaughy, DiClemente, Prochaska y Velicer, 1989), auto informe de tipo likert que consta de 32 ítems en función de los cuatro estadios de cambio: precontemplación, contemplación, acción y mantenimiento. Las alternativas de respuesta valoran los ítems en una escala de cinco puntos desde fuertemente en desacuerdo (1) hasta sumamente de acuerdo (5). EI URICA permite categorizar a los pacientes en el estadio de cambio con la calificación T más alta (Finnell, 2003), y en caso de que las puntuaciones $T$ sean iguales en diversos estadios se categoriza al paciente en el estadio más avanzado (Tsoh, 1995).

El presente estudio sirvió como piloto para adaptar los ítems del URICA a nuestra realidad. Para revisar el grado de error de medición se examino la confiabilidad mediante el método de consistencia interna de Kuder-Richardson; relacionando las varianzas de cada uno de los ítems con la varianza total de cada una de las subescalas aplicada antes y después de las sesiones de estimulación motivacional. Dicho análisis no debe ser afectado por la acción de la terapia, tal como se comprueba con los coeficientes hallados que se reproducen en la tabla 1 .

Tabla 1. Coeficientes de confiabilidad de las Subescalas del URICA por el método de la consistencia interna.

\begin{tabular}{lcccc}
\hline Sub escalas & $\begin{array}{c}\text { K20 del } \\
\text { MO I }\end{array}$ & $\begin{array}{c}\text { Error de } \\
\text { medición }\end{array}$ & $\begin{array}{c}\text { K20 del } \\
\text { M0 2 }\end{array}$ & $\begin{array}{c}\text { Error de } \\
\text { medición }\end{array}$ \\
\hline Precontemplación & 0.83 & Baja & 0,83 & Baja \\
Contemplación & 0.83 & Baja & 0,86 & Baja \\
Acción & 0.86 & Baja & 0,84 & Baja \\
Mantenimiento & 0.89 & Baja & 0,97 & Muy Baja \\
\hline
\end{tabular}

De la tabla 2 se desprende que los coeficientes hallados están dentro de los márgenes de error permitidos en estudios psicológicos, debido a la diversidad de variables individuales que actúan como fuentes de error. Sin embargo, siempre deberemos buscar que los errores de medición sean menores de 0,80 para no superar a un error del 11\%. Respecto de la validez de la escala, nos apoyamos en el criterio de construcción por el que esperamos que las personas en el estadio de precontemplación no presenten conciencia del hecho adictivo ni interés por superar sus problemas; por tanto, es de suponer correlaciones negativas con los estadios de contemplación, acción y mantenimien- to. No obstante, en la medida en que la persona pase al estadio de contemplación hay que esperar modificaciones en la dirección de la correlación, debiendo ser éstas directas positivas; vale decir, que al aumentar la motivación contenida en la contemplación se incremente el impulso para la acción de cambio y el mantenimiento, tal como hemos encontrado y que se puede ver en la tabla 2, confirmando la validez de la escala.

Tabla 2. Ínter correlaciones e ínter estadios de la escala del URICA obtenida con una muestra de 128 participantes

\begin{tabular}{lccc}
\hline & Precontemplación & Contemplación & Acción \\
\hline Precontemplación & 1 & & \\
Contemplación & $-0,45^{* *}$ & 1 & \\
Acción & $-0,21^{* *}$ & $0,66^{* *}$ & 1 \\
Mantenimiento & $-0,14$ & $0,40^{* *}$ & $0,49^{* *}$ \\
\hline
\end{tabular}

$\left.{ }^{* *}\right) p<0.01$

La satisfacción de los consultantes con el programa se evalúo con el Cuestionario de Satisfacción con el Tratamiento -CST (Larsen, Attkinsson, Hargreaves, Nguyen, Roberts y Stegner, 1979) que consta de ocho ítems relacionados con la calidad de la atención terapéutica y la intensidad de la ayuda recibida; en último término, como su nombre lo indica, con la satisfacción respecto al tratamiento. Dicho cuestionario ha sido utilizado en diversas poblaciones y grupos étnicos, acreditando una gran consistencia interna y capacidad discriminante (Fischer y Corcoran, 1996). La versión en lengua castellana (Roberts y Attkinsson, 1984), aunque realizada fuera de nuestro país, parece comportarse de igual manera que la versión inglesa, cuando una y otra son correlativamente utilizadas en población hispana o angloparlante (Roberts, Attkinsson y Mendias, 1984). El análisis de los resultados obtenidos en el presente estudio utilizando el CST mediante un alfa de Cronbach evidenció una alta consistencia interna de 0,86.

Para realizar el seguimiento de los casos se elaboro una encuesta simple (Encuesta de Seguimiento de Casos -ESC) de ocho ítems.

\section{Estructura de las sesiones de consejo psicológico y motivacional}

La tabla 3 detalla la estructura de las sesiones del programa (Rojas, 2006). 
Tabla 3. Estructura de las sesiones de consejo psicológico y motivación

\begin{tabular}{|c|c|c|}
\hline $\mathbf{N}^{\circ}$ & SESIONES & DESCRIPCIÓN \\
\hline I & Acogida y Apertura & $\begin{array}{l}\text { Información sobre las sesiones y se le delega responsabilidades. Se descarta trastornos de per- } \\
\text { sonalidad, comportamental o psiquiátrico. Se controlan estados de ánimo, pudiéndose aplicar } \\
\text { terapia farmacológica en caso sea necesario; se formula un plan de trabajo. }\end{array}$ \\
\hline$\|$ & Técnicas Basadas en la Información & $\begin{array}{l}\text { Se proporciona información seleccionada, se le instruye sobre el comportamiento sintomático y } \\
\text { se le da instrucciones de cómo actuar. }\end{array}$ \\
\hline III & Afianzamiento de la Motivación & $\begin{array}{l}\text { Activar la motivación intrínseca generando un cambio de actitud frente al consumo; se le enseña a } \\
\text { reconocer, evitar y afrontar los pensamientos asociados al consumo e identificar la ambivalencia. }\end{array}$ \\
\hline IV & Habilidades de Rechazo & $\begin{array}{l}\text { Se le entrena en respuestas de rechazo al ofrecimiento de drogas. Se establecen estrategias para } \\
\text { romper los contactos con los proveedores. }\end{array}$ \\
\hline V & Control de Estímulos & $\begin{array}{l}\text { Eliminar los estímulos que estén provocando o impidiendo la ejecución de una conducta determi- } \\
\text { nada y los estímulos relacionados al consumo a través de técnicas de autocontrol. }\end{array}$ \\
\hline VI & Pensamientos Automáticos & $\begin{array}{l}\text { Se intenta que separe situaciones problemáticas de sus pensamientos y sus emociones, de tal } \\
\text { forma que pueda identificar los pensamientos de alta tensión y los pensamientos de consumo. }\end{array}$ \\
\hline VII & Programa de Autocontrol & $\begin{array}{l}\text { Disminuir conductas autodestructivas, se enseñan técnicas de cambio conductual mediante la } \\
\text { focalización en los antecedentes y en las consecuencias; así como en la importancia del auto- } \\
\text { control. }\end{array}$ \\
\hline
\end{tabular}

\section{Procedimiento}

En la primera sesión (acogida) el consejero recolectó información del consultante y/o familiar acompañante utilizando la Ficha de Atención (FdA) y aplicó también al consultante la Escala de Evaluación para el Cambio de la Universidad de Rhode Island (URICA).

Luego de tres semanas -equivalentes a tres sesiones- antes de la derivación-, se volvió a aplicar el URICA con la finalidad de conocer los cambios en los estadios motivacionales después de la aplicación del programa (post-test) y el Cuestionario de Satisfacción con el Tratamiento (CST) para evaluar la percepción y satisfacción con relación al servicio brindado. El seguimiento fue personalizado y telefónico (Encuesta de Seguimiento de Casos, ESC)

Previamente los participantes completaron un formulario de consentimiento informado (mayores de edad) o asentimiento informado (menores de edad). Los formularios utilizados fueron revisados y aprobados por el Comité Institucional de Ética de la Universidad Peruana Cayetano Heredia (UPCH).

\section{Análisis de información}

Los datos recolectados y codificados fueron sometidos a tratamiento estadístico en el programa estadís- tico Statistical Package of Social Science-SPSS 11.0 (Pardo y Ruiz, 2002).

\section{RESULTADOS}

\section{Población y aspectos sociodemográficos}

De los 358 consultantes atendidos en este período, sólo 200 cumplían con los requisitos de inclusión para participar en el estudio; de esta submuestra de consultantes, únicamente 128 culminaron con las sesiones de orientación, consejería y motivación, siendo 72 los consultantes que abandonaron el programa antes de culminar con dichas sesiones (tabla 4).

\section{Tabla 4. Total de consultantes atendidos}

\begin{tabular}{|l|c|}
\hline & $n$ \\
\hline Consultantes atendidos en el programa (febrero-agosto 2005).* & 358 \\
\hline Consultantes incluidos en el estudio. & 200 \\
\hline Consultantes que abandonaron antes de culminar las sesiones. & 72 \\
\hline Consultantes que culminaron las sesiones. & 128 \\
\hline
\end{tabular}

* El 44,1\% de los consultantes no cumplían con los criterios de inclusión (policonsumo, trastornos psiquiátricos, intoxicación por SPAs, adicciones no tóxicas, otros problemas) 
Tabla 5. Adherencia y abandono

\begin{tabular}{|c|c|c|c|c|}
\hline \multirow{2}{*}{ VARIABLES } & \multicolumn{2}{|c|}{ ADHERENCIA } & \multicolumn{2}{|c|}{ ABANDONO } \\
\hline & $\mathbf{N}$ & $\%$ & $\mathbf{n}$ & $\%$ \\
\hline \multicolumn{5}{|l|}{ EDAD } \\
\hline Menor de 18 años & 28 & $21,9 \%$ & 46 & $63,9 \%$ \\
\hline De 18 a 23 años & 56 & $43,8 \%$ & 8 & $11,1 \%$ \\
\hline De 24 a 29 años & 16 & $12,5 \%$ & 5 & $6,9 \%$ \\
\hline De 30 a 35 años & 19 & $14,8 \%$ & 5 & $6,9 \%$ \\
\hline Mayor de 35 años & 9 & $7,0 \%$ & 8 & $11,1 \%$ \\
\hline TOTAL & 128 & $100.0 \%$ & 72 & $100.0 \%$ \\
\hline \multicolumn{5}{|l|}{ DROGA PROBLEMA } \\
\hline Marihuana & 59 & $46,1 \%$ & 38 & $52,8 \%$ \\
\hline Cocaína & 27 & $21,1 \%$ & 17 & $23,6 \%$ \\
\hline Alcohol & 22 & $17,2 \%$ & 10 & $13,9 \%$ \\
\hline Pasta básica de cocaína & 19 & $14,8 \%$ & 7 & $9,7 \%$ \\
\hline Inhalantes & 1 & $0,8 \%$ & 0 & $0,0 \%$ \\
\hline TOTAL & 128 & $100.0 \%$ & 72 & $100.0 \%$ \\
\hline
\end{tabular}

Tabla 6. Características socio demográficas de la muestra $(n=128)$

\begin{tabular}{|c|c|c|}
\hline VARIABLE & $\mathrm{N}$ & $\%$ \\
\hline \multicolumn{3}{|l|}{ Edad } \\
\hline Menor de 18 años & 28 & $21,9 \%$ \\
\hline De 18 a 23 años & 56 & $43,8 \%$ \\
\hline De 24 a 29 años & 16 & $12,5 \%$ \\
\hline De 30 a 35 años & 19 & $14,8 \%$ \\
\hline Mayor de 35 años & 9 & $7,0 \%$ \\
\hline \multicolumn{3}{|l|}{ Estado Civil } \\
\hline Soltero & 101 & $78,9 \%$ \\
\hline Casado & 18 & $14,1 \%$ \\
\hline Divorciado o Separado & 5 & $3,9 \%$ \\
\hline Conviviente & 4 & $3,1 \%$ \\
\hline \multicolumn{3}{|l|}{ Nivel de Instrucción } \\
\hline Secundaria incompleta & 5 & $3,9 \%$ \\
\hline Secundaria completa & 50 & $39,1 \%$ \\
\hline Técnica incompleta & 11 & $8,6 \%$ \\
\hline Técnica completa & 11 & $8,6 \%$ \\
\hline Superior universitaria incompleta & 22 & $17,2 \%$ \\
\hline Superior universitaria completa & 29 & $22,7 \%$ \\
\hline \multicolumn{3}{|l|}{ Ocupación } \\
\hline Estudiante & 49 & $38,3 \%$ \\
\hline Desempleado & 46 & $35,9 \%$ \\
\hline Empleado & 20 & $15,6 \%$ \\
\hline Profesional Universitario & 6 & $4,7 \%$ \\
\hline Comerciante & 3 & $2,3 \%$ \\
\hline Profesional Técnico & 3 & $2,3 \%$ \\
\hline Ama de Casa & 1 & $0,8 \%$ \\
\hline
\end{tabular}

En la tabla 5 podemos observar datos referidos a los niveles de adherencia y abandono del programa. Siendo los consultantes de 18 a 23 años, quienes tuvieron mayor adherencia al programa, mientras que los participantes de 24 a 29 años y los mayores de 35 años presentaron las tasas más bajas de adherencia al programa. Por otro lado, se evidencio que los consul- tantes menores de 18 años fueron quienes en mayor número desertaron del programa. La misma tabla muestra que fueron los consumidores de marihuana quienes tuvieron mayor adherencia al programa.

La tabla 6 muestra las características socio demográficas de los consultantes. El rango de edad de la muestra se encuentra entre los 15 a 51 años, siendo 
el intervalo de 18 a 23 años donde se concentra la mayoría de los consultantes (43,8\%). En cuanto al estado civil destaca que un 78,9\% de los consultantes son solteros/as y con educación secundaria completa $(39,1 \%)$ siendo la mayoría estudiantes (38,3\%).

\section{Características del consumo de SPA}

En la tabla 7 se observa que casi la mitad de los consultantes tienen problemas de consumo de marihuana $(46,1 \%)$ asimismo poco más de la cuarta parte de la muestra $(33,6 \%)$ tiene de uno a dos años de consumo. Por otro lado, respecto de la frecuencia de consumo de la droga problema, se aprecia que el $59,4 \%$ consume entre 2 a 3 veces a la semana.

Tabla 7. Patrones de consumo $(n=128)$

\begin{tabular}{|l|c|c|}
\hline VARIABLES & N & $\%$ \\
\hline Droga Problema & 59 & $46,1 \%$ \\
\hline Marihuana & 27 & $21,1 \%$ \\
\hline Cocaína & 22 & $17,2 \%$ \\
\hline Alcohol & 19 & $14,8 \%$ \\
\hline Pasta básica de cocaína & 1 & $0,8 \%$ \\
\hline Inhalantes & 7 & $5,5 \%$ \\
\hline Tiempo de Consumo & 3 & $2,3 \%$ \\
\hline Menos de 6 meses & 43 & $33,6 \%$ \\
\hline De 6 meses a un año & 37 & $28,9 \%$ \\
\hline De 1 a 2 años & 38 & $29,7 \%$ \\
\hline De 2 a 4 años & 3 & $2,3 \%$ \\
\hline Más de 4 años & 14 & $10,9 \%$ \\
\hline Frecuencia de Consumo & 76 & $59,4 \%$ \\
\hline Una vez al mes & 12 & $9,4 \%$ \\
\hline Una vez cada semana & 23 & $18,0 \%$ \\
\hline 2 ó 3 veces a la semana & \\
\hline 4 ó 5 veces a la semana & Todos los días & \\
\hline \multicolumn{2}{|l|}{} \\
\hline
\end{tabular}

Aspectos de la evaluación de la dinámica motivacional de los participantes antes y después de las intervenciones motivacionales, según estadio de cambio

\section{Efectos de las intervenciones motivacionales en la evaluación para el cambio}

Por la naturaleza del estudio y de sus participantes, no ha sido posible utilizar un grupo control. Así, cada uno de los consultantes actuó a la vez como su propio control, por lo que los hemos evaluado al empezar y al concluir las intervenciones motivacionales.
Los datos obtenidos mediante el URICA corresponden a escalas ordinales y, además, al efectuarse la prueba de bondad de ajuste conforme a la normalidad estadística con la ecuación de Kolmogorov-Smirnov encontramos valores $Z$ con valores $P$ mayores al 0,05, de conformidad con la hipótesis nula, por lo que resultó conveniente contrastar los efectos mediante estadísticos no paramétricos utilizando la prueba de Mac Nemar para la evaluación de los cambios.

Para los efectos, cada participante se contrasto consigo mismo siempre que cumpliera el criterio del cambio, es decir, pasar de un estadio a otro, no sólo en dirección positiva (avance) sino inclusive en dirección negativa (retroceso). El análisis se efectuó para el grupo en general y, para el estudio analítico de los detalles, los cálculos se realizaron en función del diagnóstico; es decir, con los participantes que en la aplicación de entrada fueron diagnosticados como pertenecientes al estadio de precontemplación, contemplación, acción y mantenimiento.

Para efectos de observación del cambio se conceptualizaron como estadios negativos a los dos primeros (precontemplación y contemplación) y positivos a los dos siguientes (acción y mantenimiento). La razón básica para ello parte del significado implícito en cada uno de ellos; de esta manera, la observación o el registro del cambio siempre advertirá si el participante ha pasado de un estadio hacia otro en cualquiera de sus direcciones o, en su defecto, si permanece sin modificación significativa.

\section{Análisis del cambio en el grupo en general}

Se encontró que de los 128 participantes, 25 fueron en un inicio diagnosticados en un estadio superior y después de las sesiones motivacionales retrocedieron. En tanto, 44 participantes empezaron en un estadio menor y luego cambiaron positivamente; 23 iniciaron en estadios positivos y continuaron sin cambio, en tanto que 36 comenzaron en el estadio negativo y se mantuvieron en el mismo. Los cálculos efectuados dieron un valor de cambio de 4,94, que es significativo al 0,05 de margen de error; por tanto, podemos afirmar que las intervenciones motivacionales han tenido resultados positivos.

Asimismo se observo que para un grado de libertad el valor teórico es 3,84 al 0,05 de margen de error; así, la probabilidad hallada es menor del 0,05, lo que indica que la razón de cambios encontrados es significativa hacia la dirección positiva. Por ello, las sesiones motivacionales, en términos generales, han dado el resultado esperado. 


\section{Análisis del cambio en los participantes con diag- nóstico de precontemplación y contemplación}

Es conveniente aclarar que en este grupo se ha incluido a los participantes con diagnóstico de precontemplación, pues en la muestra total sólo fueron dos; ellos están dentro del criterio señalado, es decir, dentro de la dirección negativa.

En el análisis correspondiente se encontró que ninguno de los participantes retrocedió, pero sí encontramos que 35 de los 75 consultantes empezaron en el estadio negativo y se mantienen; sólo con un caso ha ocurrido en el estadio positivo. Lo positivo es que 39 de los evaluados han pasado de estadios inferiores a superiores.

Siendo el cambio muy significativo aun para el 0,001 de margen de error. Por ende, cuando los participantes involucrados en su problema hacen conciencia del mismo con una ayuda especializada, éstos pueden dar el paso hacia la acción comprometedora con el cambio.

\section{Análisis del cambio en los participantes con el diagnóstico de pertenecer al estadio de acción}

Es esperado que los participantes situados en el estadio de acción acudan a recibir ayuda; sin embargo, un alto número de los mismos se mantuvo en este estadio: 20 de 39 participantes, esto es ligeramente algo más de la mitad (51\%). De este modo, resalta el hecho de que 13 consultantes (33\%) regresionaron al estadio anterior y sólo 5 participantes cambiaron positivamente, representando únicamente el $13 \%$. Siendo el valor encontrado de 3,06 menor al valor crítico de 3,84; por tanto, la probabilidad de ocurrencia conforme con la hipótesis nula es mayor del 0,05, lo que evidenció que en este grupo de participantes las sesiones motivacionales no dieron el resultado esperado.

\section{Análisis del cambio en los participantes con diag- nóstico en el estadio de mantenimiento}

En este grupo se esperaba que el $100 \%$ se mantenga en el estadio diagnosticado; sin embargo, se encontró que 12 de 14 participantes $(85,7 \%)$, regresionaron y sólo 2 participantes $(14,3 \%)$ se mantuvieron en el estadio de mantenimiento.

El valor crítico de 10,1 es muy significativo al 0,01 de margen de error, pero de cambio regresivo; es decir, no obstante haber superado estadios, no se pueden mantener en uno de ellos. En general, las sesiones motivacionales han producido cambios, los mismos que se deben a la demanda de los participantes para pasar a la acción y así superar el problema.
Sin embargo, cuando consiguen llegar al estadio superior, la tendencia a regresionar es significativa.

\section{Evaluación de la percepción y satisfacción del pro- grama, según los participantes del estudio}

En cuanto a los resultados de la percepción y satisfacción del servicio, el 46,9\% calificó la calidad del servicio recibido como excelente, la mitad $(50,0 \%)$ como bueno, y una minoría 3,1\% percibió que la calidad del servicio era regular. El 41,4\% manifestó que encontró en su totalidad la clase de servicio que buscaba, el $42,2 \%$ encontró en general el servicio que buscaba y un $16,4 \%$ manifestó haber encontrado parcialmente el servicio buscado.

De los consultantes encuestados, un $28,9 \%$ estaba totalmente satisfecho con el programa, pues éste había colmado sus necesidades de demanda, seguidos por más de la mitad $(55,5 \%)$ que observó que sus necesidades de ayuda en general fueron atendidas. El $15,6 \%$ de los consultantes se mostró parcialmente satisfecho; el 73,4\% indicó que recomendaría el programa, el 25,0\% probablemente recomendaría nuestro programa a algún familiar o amigo que necesite una ayuda similar, y una minoría $(1,6 \%)$ no recomendaría nuestro programa.

En cuanto a la satisfacción con el tipo de ayuda recibida un $40,6 \%$ estuvo muy conforme, seguido por un $34,4 \%$ que indicó estar considerablemente satisfecho; el $23,4 \%$ de los consultantes se encuentra bastante satisfecho con la ayuda brindada y sólo una minoría (1,6\%) manifestó estar poco conforme con la ayuda recibida. La mayoría de los consultantes $(49,2 \%)$ percibió que las sesiones recibidas en el programa le ayudaron a hacer frente más eficazmente a sus problemas, y una minoría $(7,8 \%)$ encontró poca ayuda para solucionar sus problemas en el programa. Casi la totalidad de los consultantes $(95,3 \%)$ manifestó encontrarse mucho y bastante satisfecho en general con la atención que recibió en el programa, y sólo el 4,7\% reveló hallarse en general poco satisfecho con el servicio recibido. Más de la mitad de los consultantes $(73,4 \%)$ volvería al programa en caso de necesitar nuevamente ayuda; un 24,2\% probablemente recurriría otra vez al programa, seguido por una pequeña parte de los consultantes $(2,4 \%)$ que probablemente no volvería a solicitar ayuda.

Los resultados mostraron que un $89,8 \%$ de los consultantes acudió a tratamiento después de las sesiones de orientación y consejería, siendo sólo un $10,2 \%$ no asistió a tratamiento. Asimismo, casi la totalidad de la muestra $(96,5 \%)$ asistió al centro de tratamiento y/o profesional recomendado por el programa y sólo una minoría $(3,5 \%)$ no siguió la recomendación dada por el consejero/a con relación a la derivación. En cuanto al tiempo de incursión al tratamiento luego 
de la derivación, más de la mitad de los consultantes $(74,0 \%)$ demoró menos de una semana en acercarse al centro y/o profesional recomendado, un 16,5\% demoró entre una a dos semanas, un 7,8\% tardó entre tres semanas a un mes y sólo un 1,7\% esperó más de un mes.

\section{DISCUSIÓN}

La tasa de adhesión al programa fue del 64,0\% (128 casos) siendo un 36,0\% (72 casos) el porcentaje de abandonó, proceso que tenía como criterio mínimo tres sesiones (tres semanas). Estos resultados guardan relación con las características del estadio precontemplativo (Prochaska, DiClemente y Norcross, 1992; Wilkinson y LeBreton, 1986) en la medida en que el $76,4 \%$ de los que abandonaron se ubicaron en este estadio, donde hay una ausencia de conciencia del hecho adictivo; mientras que el $23,6 \%$ si bien se habría ubicado en los estadios de contemplación, acción y mantenimiento, se podría inferir que el abandono del programa puede deberse a factores como la ambivalencia, la comorbilidad psiquiátrica, etc.

Los resultados encontrados en cuanto a la tasa de abandono sugieren que en la fase de motivación se debería facilitar el acceso a los reforzadores ajenos a las sustancias y centrarse sobre los problemas que motivaron la búsqueda de tratamiento si se pretende mantener la motivación del adicto/a en la fase motivacional. Esta tasa coincide con resultados encontrados por Battjes, Onken y Delany (1999).

Los análisis de la variable edad y droga problema en relación con la adherencia y abandono, nos muestran que un $48,8 \%$ de los participantes que concluyeron con las sesiones establecidas en el programa (128), estaba conformado por púberes, adolescentes y jóvenes. Además, se observa que el $75,0 \%$ de los 72 consultantes que abandonaron el proceso eran menores de 18 años y adolescentes/jóvenes de 18 a 23 años, deduciéndose que en este grupo tuvo baja motivación de cambio y falta de conciencia del hecho adictivo.

En los que se mantuvieron en el programa (adhesión) según droga problema, los resultados mostraron que la mayoría eran consumidores de marihuana $(46,1 \%)$, pues es sabido que los usuarios de esta sustancia suelen minimizar los riesgos del consumo hasta llegar a apologizar su uso en algunos casos. Estos resultados coinciden con lo encontrado por otros investigadores (Miller y Rollnick, 2002; Rojas, 2005; Sampl y Kadden, 2004).

Consideramos que los estadios de motivación podrían guardar cierta relación con los tipos de SPA consumidas por los participantes del estudio y, por consiguiente, también con la efectividad de tratamiento en función de adherencia, abandono y recaídas (Da Silva y cols,, 2003). Si bien se carece de información específica, al parecer los niveles motivacionales en dependencia de sustancias cocaínicas, opiáceos, etc., no necesariamente son los mismos que los que presenta la dependencia cannábica o SPA aceptadas socialmente como el tabaco y el alcohol.

En cuanto a las diferencias en los estadios de cambio antes y después de las intervenciones motivacionales, el análisis de los resultados mostraron un valor de cambio de 4,94 que es significativo al 0,05 de margen de error. Estos resultados nos demuestran que las intervenciones motivacionales brindadas por el programa, en términos generales, han tenido resultados positivos.

En otro aspecto, el análisis de los estadios de cambio en los participantes que iniciaron las sesiones motivacionales en precontemplación y contemplación evidenció cambios significativos. Por ello se puede pronosticar que las personas involucradas con el consumo de SPA asuman conciencia del hecho adictivo como consecuencia de las sesiones de estimulación motivacional y, consecuentemente, para dar el paso hacia el estadio de acción, en el cual se consolidará su compromiso para el cambio.

Se esperaba también que los participantes que iniciaban las sesiones motivacionales en el estadio de acción acudieran en busca de ayuda especializada; sin embargo, más de la mitad (51\%) se mantiene en este estadio luego de las sesiones motivacionales; el 33\% regresiona al estadio anterior y sólo 13\% avanza positivamente.

Estos resultados sugieren que los participantes que se inician en el estadio de acción, en las sesiones motivacionales no dan el resultado esperado. Ello confirmaría que un número indeterminado de consumidores podrían solicitar ayuda; sin embargo, su intención de cambio estaría supeditada a refuerzos y otros aspectos que están fuera del contexto del tratamiento (Da Silva, 2005a; Pereira de Oliveira y Malbergier, 2003; Rumpf, Hapke y John, 1998; Santos y cols,, 2001).

En aquellos que llegaron en el estadio de mantenimiento, se encontró que el $85 \%$ regresionó a otras fases y sólo el 14\% se mantuvo en tal estadio, siendo lo esperado que el $100 \%$ de los participantes se mantuviera en el mismo estadio en que inició las sesiones. En otras palabras este grupo, habiendo superado estadios, no puede mantenerse en determinada fase.

Los avances y retrocesos en el tránsito de los cambios de los estadios motivacionales de este grupo de estudio - antes y después de las sesiones-guardan relación con lo encontrado en diversos estudios donde 
se evidencia que no hay un progreso lineal a través de los estadios de cambio y conceptualizan éstos mediante un modelo en espiral o circunflejo (Casas y Gossop, 1993; Marlatt y Gordon, 1985; Miller y Rollnick, 2002; Prochaska y DiClemente, 1986; Prochaska, Velicer, Guadagnoli, Rossi y DiClemente, 1991).

En la escala de mantenimiento, los participantes se ubican con fuerte tendencia a la regresión antes que a sostenerse en el tiempo hasta encontrar las formas más apropiadas de un afrontamiento sostenible. En el estudio se nota que es la etapa de mayor complicación y conflicto en el individuo, puesto que experimentan que el andar, costoso y de mucho sacrificio, representa un riesgo de derrumbarse, y en el común de los casos se regresiona. Este dato justifica y merece revisar los pasos a seguir con el fin de reducir la regresión y por lo menos conseguir la sostenibilidad.

Los resultados de percepción y satisfacción reflejan una buena percepción y alta satisfacción lo que confirman que las intervenciones de Lugar de Escucha-CEDRO es una alternativa terapéutica importante como preámbulo al tratamiento intensivo (Miller y Rollnick, 2002), deducciones que guardan relación con la primera evaluación de satisfacción del mismo programa llevada a cabo por Rojas y Espinoza (2003).

Independientemente de los resultados obtenidos sobre la satisfacción del servicio, sean favorables o desfavorables, estos insumos deben constituirse en el eje principal para modificaciones futuras sobre la estructura de "Lugar de Escucha", considerando que la drogodependencia es un fenómeno dinámico y multifactorial; incluyendo el adecuado entrenamiento y capacitación de los consejeros (Bartu, 1996; Rohrer, Thomas y Yasenchak, 1992).

En cuanto al grado de adherencia, en la evaluación del seguimiento de los casos examinados (intervención pre y postest), se encontró que el 89,8\% de los casos (simultáneamente a las sesiones brindadas en el programa) fue derivado. Estos acudieron a algún centro de tratamiento, reflejando que este grupo mayoritario guardó coherencia e intención de involucrarse con el tratamiento. Igualmente, se encontró que un 73,9\% asistió a la alternativa recomendada dentro de la primera semana de culminadas las sesiones en el programa. Pero también se determinó que del 89,6\% de los casos (si bien acudieron a un determinado centro de atención), el 96,5\% fue al centro recomendado por el psicoterapeuta y un 3,5\% no asistió al centro de ayuda indicado.

Ello podría obedecer a factores como la gran distancia de las citas otorgadas a los pacientes que genera desaliento, así como en los estados emocionales cambiantes y de ambivalencia de los drogodependientes
Como balance global de los resultados obtenidos en la satisfacción con el servicio brindado, se podría decir que el programa cumple sus objetivos en cuanto a la derivación o referencia dentro del marco del consejo psicológico y las entrevistas motivacionales.

Cabe destacar que al utilizarse en el estudio un diseño pretest - postest de un solo grupo, este limita y tiene implicaciones en relación a la validez de los resultados. Sin embargo lo encontrado en los resultados de la investigación son destacables debido a la singularidad del programa "Lugar de Escucha" de CEDRO, que tiene como finalidad servir de puente entre la demanda de ayuda y las distintas alternativas de tratamiento.

Es importante enfatizar en el nivel motivacional logrado en las etapas iníciales de la demanda de ayuda, los mismos que deberían ser retroalimentados y afianzados en los respectivos centros donde se derivan los casos. Por lo que es necesario mantener y reforzar la coordinación sistemática entre nuestro programa y los centros de derivación, tal como lo sugieren Rumpf, Hapke y John (1998).

\section{CONCLUSIONES}

Las intervenciones motivacionales y de consejo psicológico del programa "Lugar de Escucha" de CEDRO sostienen y afianzan la motivación hacia el cambio de actitud del paciente respecto del consumo de SPA y de su motivación.

Las intervenciones de este programa son más eficaces cuando los participantes inician el proceso en los estadios de precontemplación y contemplación.

Las intervenciones del programa no son muy eficaces con pacientes que inician en un estadio de acción o mantenimiento, siendo necesaria en pacientes atendidos inicialmente en esta etapa la derivación inmediata a alternativas más compatibles con su estado motivacional. Probablemente a programas de psicoterapia profunda.

El grado de adherencia del programa es mayor en relación con la tasa de abandono.

La mayoría de consultantes que abandonó el programa se encontraba en el estadio precontemplativo.

La intervención de estimulación motivacional con afronte cognitivo-conductual y humanista, contribuye en el "enganche terapéutico" hacia las distintas formas de tratamiento desadictivo.

En lo que se refiere a las metas del programa, el servicio cumple sus objetivos generales y específicos. 
Los consultantes atendidos en el programa se muestran en su mayoría satisfechos con el servicio recibido.

\section{AGRADECIMIENTOS}

Al Programa de Prevención del Consumo de Drogas y de Rehabilitación de Toxicómanos DEVIDACooperación Técnica de Bélgica, quienes financiaron la presente investigación.

\section{REFERENCIAS}

Alfonzo, L. y Madrigal, E. (2000). La dependencia de las drogas y su tratamiento. Una experiencia en las Américas. Washington D.C.: CICAD/OEA-OPS/OMS.

Bartu, A. (1996). Client Satisfaction: Why Bother? Journal of Substance Use \& Misuse, 1, 20-26.

Battjes, R., Onken, L. \& Delany, P. (1999). Drug abuse treatment entry and engagement: report of meeting on treatment readiness. Journal of Clinical Psychology, 5, 643-657.

Casas, M. y Gossop, M. (1993). Recaída y prevención de recaídas. Barcelona: Ediciones en Neurociencias.

Da Silva, M. (2005a). Avaliação da motivação para mudança nos comportamentos adictivos. Trabajo presentado en el $30^{\circ}$ Congreso Interamericano de Psicología- Hacia una psicologia sin fronteras, Junio, Buenos Aires.

Da Silva, M. (2005b). Avaliação e Intervenção Breve em Adolescentes usuários de drogas. Revista Brasilera de Terapias Cognitivas, 1, 69-74.

Da Silva, M., Laranjeira, R., Brasil, R., Leal, R. \& Di Giorgio, D. (2003). Estudo dos estágios motivacionais em sujeitos adultos dependentes do álcool. Psicología: Reflexão e Crítica, 16, 265-270.

Finnell, D. (2003). Use of the transtheoretical model for individuals with co-occurring disorders. Community Mental Health Journal, 39, 3-13.

Fischer, J. \& Corcoran, K. (1996). Measures for Clinical Practice. Nueva York: The Free Press.

Kerlinger, F. (1994). Investigación del comportamiento. México D. F.: Mc Graw- Hill.

Larsen, D.L., Attkinsson, CC., Hargreaves, W.A., LeVois, M., Nguyen, T.D., Roberts, R.E. \& Stegner, B. (1979). Assessment of client / patient satisfaction: development of a general scale. Evaluation and program planning, 2, 197-207.

Marlatt, G. A. \& Gordon, J. R. (1985). Relapse Prevention. Nueva York: The Guilford Press.

McConnaughy, E., DiClemente, C., Prochaska, J. \& Velicer, W. (1989). Stages of change in psychotherapy: A follow-up report. Psychotherapy: Theory, Research and Practice, 26, 494-503.
McConnaughy, E., Prochaska, J. O. \& Velicer, W. (1983). Stages of change in psychotherapy: Measurement and sample profiles. Psychotherapy: Theory, Research and Practice, 20, 368-375.

Miller, W. R. \& Rollnick, S. (2002). Motivational Interviewing: Preparing People for Change. Nueva York: Guilford Press.

Pardo, A. y Ruiz, M. A. (2002). SPSS 11.0. Guía para el análisis de datos. Madrid: McGraw-Hill.

Pereira de Oliveira, H. \& Malbergier, A. (2003). Avaliação da motivação para tratamento em pacientes dependentes de álcohol que procuram um serviço especializado. Revista Brasilera de Psiquiatría, 25 (1), 5-10.

Prochaska, J. O. \& DiClemente, C. C. (1986). Toward a Comprehensive Model of Change. En W. R. Miller \& N. Heather (Eds.), Treating Addictive Behavior. Processes of Change (pp. 3-27). Nueva York, USA: Plenun Publishing Corporation.

Prochaska, J. O., DiClemente, C. C. \& Norcross, J. C. (1992). In search of how people change: Applications to the addictive behavior. American Psychologist, 47, 1102-1114.

Prochaska, J. O., Velicer, W. F., Guadagnoli, E., Rossi, J. S. \& DiClemente, C. C. (1991). Patterns of change: Dinamic typology applied to smoking cessation. Multivariate Behavior Research, 26, 83-107.

Roberts, R. \& Attkinsson, C. (1984). Assessing client satisfaction among hispanics. Evaluation and Program Planning, 6, 401-413.

Roberts, R., Attkinsson, C. \& Mendias, R. (1984). Assessing the client satisfaction questionnaire in English and Spanish. Hispanic Journal Behaviour Sciences, 6, 385-395.

Rohrer, J., Thomas, M. \& Yasenchak, A. (1992). Client perceptions of the ideal addictions counsellor. The International Journal of the Addictions, 27, 727-733.

Rojas, M. y Espinoza, L. S. (2003). Evaluación de la percepción y satisfacción de las intervenciones de consejería del programa "Lugar de Escucha» de CEDRO. Manuscrito no publicado.

Rojas, M. (2005). Consumo de drogas psicoactivas en un colectivo de púberes y adolescentes. Pautas y tendencias. Lima: ONUDD-CEDRO.

Rojas, M. (2006). Manual de consejo psicológico y sesiones motivacionales en casos de drogodependencias: Estructura de las sesiones y metodología. Tomo II. Lima: CONCYTEC/CEDRO

Rojas, M., Giraldo, P. y Montes, C. (2001). Consejería telefónica, consejo psicológico e intervención en crisis. Lima: CEDRO.

Rumpf, H., Hapke, U. \& John, U. (1998). Previous help seeking and motivation to change drinking behaviour in alcohol-dependent general hospital patients. General Hospital Psychiatry, 20, 115-119. 
Sampl, S. y Kadden, R. (2004). Terapia de estimulación motivacional y cognitivo-conductual para adolescentes adictos a marihuana. Barcelona: Ars Médica.

Santos, P., González, G., Fons, M. R., Forcada, R. y Zamorano, C. (2001). Estadios y procesos de cambio en drogodependientes en tratamiento ambulatorio. Adicciones, 13, 147-152.

Tsoh, J. Y. (1995). Stages of the Change, Exits, and the Result in the Treatment of the Abuse of the Substance.
Tesis Doctoral no publicada, University of the RhodeIsland, Kingston.

Wilkinson, D. A. \& LeBreton, S. (1986). Early Indications of Treatment Outcome in Multiple Drug Users. En W. R. Miller \& N. Heather (Eds.), Treating Addictive Behavior. Processes of Change (pp. 239-261). Nueva York, USA: Plenum Publishing Corporation. 